Beatriz Talibertida Costa Porto ${ }^{1}$

Heuzabet Salomão Abdalla Ayrosa Ribero ${ }^{2}$

Maria Antonieta Longo Galvão

VANeSSA GozZo Sekula ${ }^{4}$

JOSÉ MENDES ALDRIGUI

Paulo Augusto Ayrosa Ribero ${ }^{6}$

Artigo Original

\author{
Palavras-chave \\ Endometriose/cirurgia \\ Endometriose/classificação \\ Endometriose/patologia \\ Histologia \\ Qualidade de vida \\ Questionários \\ Keywords \\ Endometriosis/surgery \\ Endometriosis/classification \\ Endometriosis/pathology \\ Histology \\ Quality of life \\ Questionnaires
}

\section{Classificação histológica e qualidade de vida em mulheres portadoras de endometriose}

\author{
Histological classification and quality of life in women \\ with endometriosis
}

\section{Resumo}

OBJETIVO: Avaliar a relação entre a classificação histológica em pacientes operadas de endometriose e a qualidade de vida. MÉTODOS: Estudo observacional transversal, com avaliação de 32 biópsias de intestino, peritônio e ligamento uterossacro em 40 mulheres portadoras de endometriose profunda. Para a análise da qualidade de vida (QV) foi aplicado o questionário SF-36 antes da cirurgia e após 6 e 12 meses. As biópsias foram classificadas histologicamente em estromal puro (EP), glandular diferenciada (GD), indiferenciada (G) e mista (GM), sendo que ficaram na amostra apenas o $G$ e e GM, relacionadas aos oito domínios do SF-36. RESULTADOS: Observamos a seguinte distribuição de acordo com o tipo histológico: no peritônio 63\% Gl e 22\% GM; no intestino 19\% Gl e 24\% G e no uterossacro 4 1\% Gl e 35\% GM. Quanto à QV e à classificação histológica, a evolução dos casos com implante no intestino mostrou que apenas o tipo GM apresentou melhora de $\mathrm{O}$ a 6 meses no aspecto social e no aspecto emocional. Ainda entre esses casos, o $\mathrm{Gl}$ mostrou que os domínios estado geral da saúde $(p=0,01)$ e aspecto social $(p=0,04)$ têm relação significativa com a melhora da QV de 0 a 6 meses e uma tendência à melhora do estado geral da saúde de 0 a 12 meses. Quanto à dor $(p=0,06)$ e ao aspecto emocional ( $p=0,05)$, observamos tendência à melhora na QV de 0 a 6 meses e na capacidade vital $(p=0,1)$ de $\mathrm{O}$ a 6 e de $\mathrm{O}$ a 12 meses. No que se refere ao aspecto emocional, foi observado que entre as pacientes com tipo histológico Gl, diferente do GM, não houve evolução favorável de 0 a 6 meses. No uterossacro não observamos relações significativas entre tipo histológico e QV. CONCLUSÃO: A melhora da QV em mulheres submetidas ao tratamento cirúrgico laparoscópico de endometriose profunda apresenta associação com grau de diferenciação histológica. Apenas as pacientes com endometriose classificada como indiferenciada e com lesões no peritônio mostraram melhora da QV após a cirurgia.

\section{Abstract}

PURPOSE: To assess the relationship between the histological classification and the quality of life of patients operated for endometriosis. METHODS: A cross-sectional observational study, with assessment of 32 biopsies of the intestine, peritoneum and uterosacral ligament from 40 women with deep endometriosis. The quality of life (QOL) was determined by applying the SF-36 questionnaire pre-operatively and at 6 and 12 months postoperatively. Biopsies were histologically classified into pure stromal (EP), glandular differentiated (GD), glandular undifferentiated (G) and mixed (GM), remaining in the sample only $G \mid$ and GM, which are related to eight domains of the SF-36. RESULTS: According to the histologic type, the following distribution was observed: peritoneum 63\% Gl and 35\% GM; intestine 19\% Gl and 24\% GM; uterosacral ligament with $41 \% \mathrm{Gl}$ and $35 \%$ GM. Regarding the QOL and the histological classification, in the intestine only GM was associated with improvement of social and emotional aspects from 0 to 6 months; the domains general health status $(p=0.01)$ and social aspect $(p=0.04)$ were significantly related to improvement of the $\mathrm{QOL}$ from $\mathrm{O}$ to 6 months, and the general health status tended to improve from $\mathrm{O}$ to 12 months. Regarding pain ( $p=0.06$ ) and the emotional aspect ( $p=0.05$ ), the $Q O L$ tended to improve from 0 to 6 months and the vital capacity ( $p=0.1$ ) improved from 0 to 6 months and from 0 to 12 months. Regarding the emotional aspect, evolution of the two histological types was not favorable for improvement in MG from 0 to 6 months. No significant relationships between histologic type and QOL were evident in the uterosacral ligament samples. CONCLUSION: Improvement in the QOL of women undergoing laparoscopic surgery for deep endometriosis is associated with the histologic grade. The peritoneal biopsy of $\mathrm{Gl}$ revealed improved QOL after surgery.
Correspondência

Beatriz Taliberti da Costa Porto Rua Cesário Mota Júnior, 112 - Vila Buarque (EP: 01221-020

São Paulo (SP), Brasil

Recebido

$10 / 02 / 2013$

Aceito com modificacọoes

$30 / 12 / 2014$
Serviço de Obstetrícia e Ginecologia da Irmandade da Santa Casa de Misericórdia de São Paulo - São Paulo (SP), Brasil.

'Departamento de Obstetrícia e Ginecologia, Faculdade de Ciências Médicas da Santa Casa de São Paulo - São Paulo (SP), Brasil. 2Departamento de Obstetrícia e Ginecologia, Faculdade de Ciências Médicas da Santa Casa de São Paulo - São Paulo (SP), Brasil; Setor de Endoscopia Ginecológica e Endométrio, Irmandade da Santa Casa de Misericórdia de São Paulo - São Paulo (SP), Brasil. 3Departamento de Patologia, Faculdade de Ciências Médicas da Santa Casa de São Paulo - São Paulo (SP), Brasil.

${ }^{4}$ Faculdade de Ciências Médicas da Santa Casa de São Paulo - São Paulo (SP), Brasil.

${ }_{5}^{5}$ Departamento de Obstetrícia e Ginecologia, Faculdade de Ciências Médicas da Santa Casa de São Paulo - São Paulo (SP), Brasil; Irmandade da Santa Casa de Misericórdia de São Paulo - São Paulo (SP), Brasil:

'Clínica de Ginecologia Cirúrgica, Departamento de Obstetrícia e Ginecologia, Faculdade de Ciências Médicas da Santa Casa de São Paulo - São Paulo (SP), Brasil; Irmandade da Santa Casa de Misericórdia de São Paulo - São Paulo (SP), Brasil.

Conflito de interesses: não há. 


\section{Introdução}

A endometriose é uma doença definida como a presença de glândula e ou estroma endometrial fora do útero, o que induz uma reação inflamatória crônica ${ }^{1,2}$. Acomete 10 a $15 \%$ das mulheres em idade fértil. Pode alcançar $16 \%$ em mulheres assintomáticas e $47 \%$ em pacientes com infertilidade ${ }^{3}$.

A característica macroscópica da lesão de endometriose pode variar na forma e coloração, essa pode ser típica, sendo caracterizada pela cor escura, ou atípica, com coloração amarela, falhas peritoneais, ou avermelhadas, indicando maior atividade da doença ${ }^{4}$.

Devido ao comportamento variável da doença, pelas dúvidas quanto à etiopatogenia, ao diagnóstico e tratamento, sua classificação se tornou importante para universalizar o entendimento dessa entidade clínica ${ }^{5}$.

A intensidade dos sintomas tem relação direta com o tamanho e a localização dos focos e o estádio, até o estádio IV, mais avançado ${ }^{6}$. Dentre os critérios usados, define-se a doença peritoneal como superficial quando a invasão atinge até $1 \mathrm{~mm}$, intermediária entre 2 e $4 \mathrm{~mm}$ e profunda se igual ou superior a $5 \mathrm{~mm}^{7}$. Uma das formas de endometriose profunda é a intestinal, entendida como a presença de glândula e/ou estroma infiltrando a camada muscular da parede intestinal ${ }^{7-9}$.

Em relação às características histológicas da endometriose, alguns autores definiram que os implantes endometriais são morfologicamente idênticos à mucosa uterina ${ }^{10}$. Outros, por sua vez, constataram que há diferenciação histológica tanto na doença superficial como na profunda, em comparação com o endométrio tópico ${ }^{7,11}$.

Buscando ampliar os conhecimentos histológicos da doença foi realizado estudo com 96 pacientes operadas por endometriose avaliando o comportamento histológico das lesões endometrióticas após o uso de Danazol ${ }^{11}$. A endometriose foi classificada como estromal e glandular diferenciada, glandular indiferenciada e mista ${ }^{11}$. Em outro estudo, foram analisadas 412 biópsias de lesões sugestivas de endometriose, a classificação morfológica e histológica da endometriose foi validada e foi sugerida possível relação do grau histológico com o prognóstico da doença ${ }^{12}$.

Em nosso meio, utilizando a mesma classificação e padronização de leitura, foi comparada a diferenciação histológica de biópsias realizadas em endometriose superficial e profunda, constatando que o tipo histológico glandular bem diferenciado predomina na lesão superficial, enquanto na profunda predomina o tipo glandular misto e indiferenciado ${ }^{13}$.

Dentre os possíveis marcadores de prognóstico de cura e melhora clínica das mulheres portadoras de endometriose, a qualidade de vida antes da cirurgia foi relatado como um fator de grande importância ${ }^{14}$. Neste estudo os pesquisadores aplicaram o questionário SF-36, amplamente conhecido na avaliação genérica da qualidade de vida.

O questionário de qualidade de vida Short Form 36 foi validado e traduzido no Brasil em 1999. Contém 36 itens em 8 domínios: (D1) capacidade funcional; (D2) aspectos físicos; (D3) dor; (D4) estado geral da saúde; (D5) vitalidade; (D6) aspectos sociais; (D7) aspectos emocionais; e (D8) saúde mental $^{15}$. Foi recentemente validado para mulheres com endometriose, mostrando ser eficaz tanto para o emprego na época do diagnóstico como para avaliação do tratamento da doença ${ }^{16}$. Em nosso serviço foi aplicado o questionário SF-36 em 40 pacientes operadas de endometriose profund $\mathrm{a}^{17}$, observando melhora significativa entre 6 e 12 meses após o tratamento ${ }^{18}$.

Por esse motivo propusemos relacionar a classificação histológica da endometriose e a qualidade de vida dessas mulheres, a fim de auxiliar tanto a classificação da doença quanto para identificar um tratamento mais eficaz com base nas características histológicas.

\section{Métodos}

Foi realizado um estudo piloto do tipo observacional transversal com base em revisão de prontuário e nova análise de lâminas. Foram revisadas as biópsias de 40 pacientes operadas de endometriose profunda entre junho de 2007 e setembro de 2008. As pacientes eram oriundas da Clínica de Endoscopia Ginecológica e Endometriose do Departamento da Irmandade da Santa Casa de Misericórdia de São Paulo.

As biópsias foram retiradas de três locais padronizados como: peritônio, ligamento uterossacro e intestino. Os tipos histológicos encontrados nesses locais foram: estromal, glandular diferenciado, glandular indiferenciado e misto.

Foram incluídas pacientes com endometriose profunda (estádios III e IV segundo a American Society for Reproductive Medicine - ASRM), com comprometimento de reto e sigmoide por endometriose (camada muscular e mais interna) e que apresentavam sinais e sintomas sugestivos de endometriose profunda: dismenorreia, dispaurenia, dor à mobilização de fundo vaginal e/ou nódulos palpáveis em fórnice vaginal posterior.

Todas as pacientes tinham ciclo menstrual regular e não podiam estar em uso de medicação hormonal (análogos do hormônio liberador de gonadotrofina- $\mathrm{GnRH}$, progestagênios e contraceptivos hormonais) na ocasião da cirurgia e nos três meses precedentes. A inclusão foi independente de raça, paridade, nível de instrução e antecedente de cirurgia prévia.

O diagnóstico pré-operatório foi feito por imagem: ressonância magnética pélvica e ecoendoscopia baixa. Todas as pacientes foram operadas de endometriose profunda, via laparoscópica, pela mesma equipe cirúrgica. Nas intervenções intestinais foi realizada ressecção segmentar, segundo a técnica padrão de nossa instituição ${ }^{19}$. 
Os fragmentos obtidos pela biópsia foram avaliados macroscópica e histologicamente segundo as normas do serviço de Anatomia Patológica da Irmandade da Santa Casa de Misericórdia de São Paulo (Figura 1), empregando-se as características da amostra descritas na Tabela 1 .

Para o diagnóstico de endometriose foram considerados os critérios histológicos: presença de tecido glandular de padrão mülleriano associado a sinais de hemorragia e fibrose, e estroma de padrão endometrioide com morfologia de qualquer período de ciclo menstrual, associado ou não a hemorragia e fibrose, com ou sem elementos glandulares. Em seguida as lesões foram classificadas morfologicamente, considerando as variantes de aspecto das estruturas endometriais ectópicas e as características próprias da doença (Figura 1) $)^{12}$.
Quanto à qualidade de vida, as pacientes foram questionadas a partir do instrumento SF- $36^{15}$ antes de serem operadas, 6 meses e 12 meses após a cirurgia.

\section{Análise estatística}

O método estatístico foi realizado para as variáveis categóricas (tipo histológico) e variáveis quantitativas (os escores dos domínios do questionário SF-36). Para a análise descritiva das variáveis qualitativas, calculamos frequências absolutas e relativas. Com o objetivo de comparar os escores dos domínios do SF-36 entre os tipos histológicos, utilizamos o teste $t$ de Student independente. Considerando-se a ausência de dados similares na literatura, após coleta de dados foi realizado cálculo do tamanho amostral, que identificou que a amostra de 15 casos nos grupos histológicos Glandular

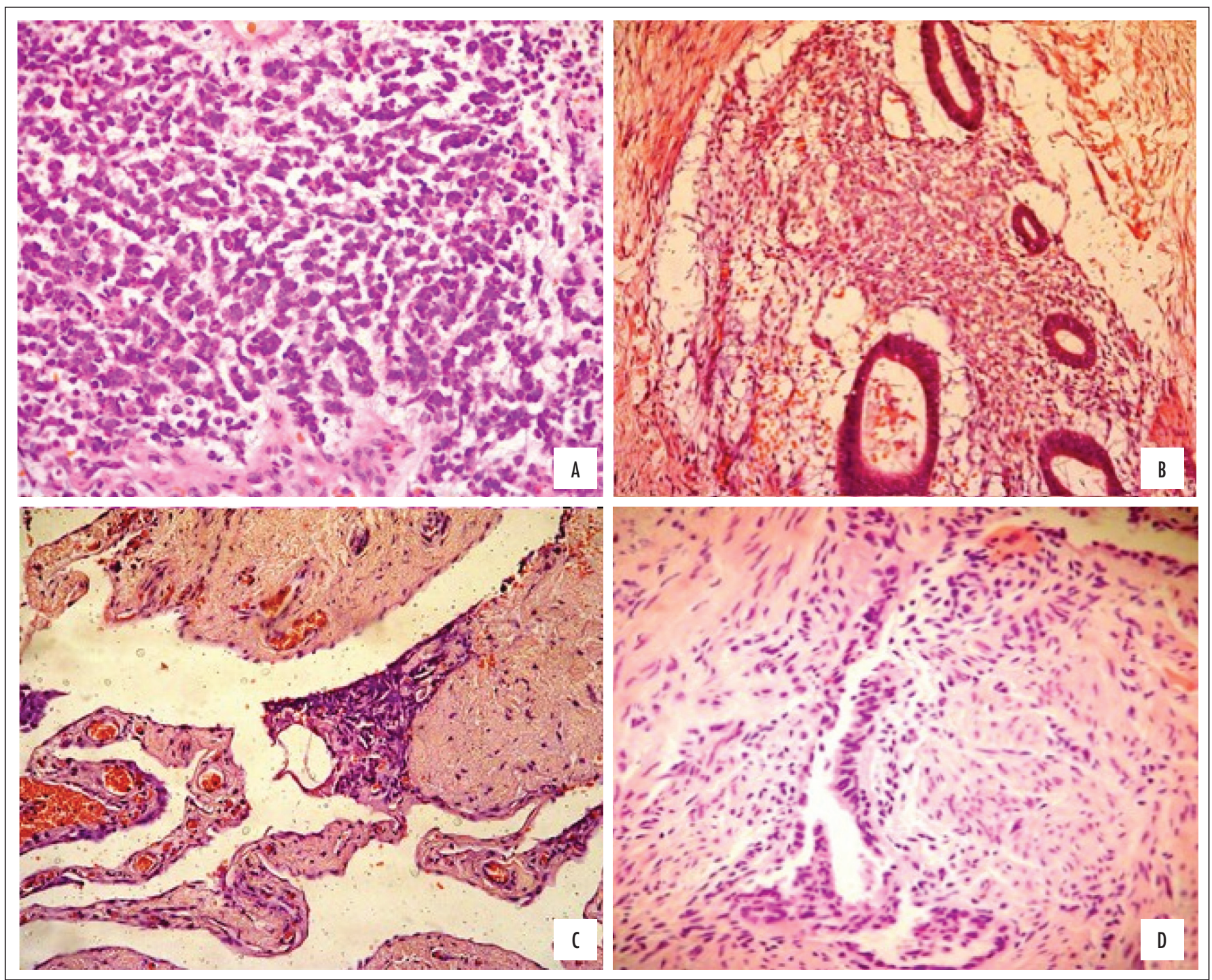

Figura 1. Lâminas mostrando padrão histológico dos diversos tipos de endometriose. (A) endometriose estromal com a presença de estroma morfologicamente similar ao endométrio tópico (400 x, HE); (B) endometriose glandular bem diferenciada caracterizada por células epiteliais de morfologia indistinguível do endométrio tópico nas diferentes fases do ciclo $(400 \mathrm{x}, \mathrm{HE})$; (C) endometriose glandular indiferenciada com epitélio glandular aplainado ou cuboidal baixo, sem correspondência com endométrio tópico, semelhante ao mesotélio do revestimento peritoneal $(400 \mathrm{x}, \mathrm{HE})$; (D) endometriose glandular mista e mostrando a presença de glândulas na mesma topografia, sendo essas de padrão diferenciado e indiferenciado (400 x, HE) 
Misto (GM) e Glandular Indiferenciado (GI) seria suficiente para detectar essa diferença, com poder de $75 \%$. O nível de significância adotado foi de $5 \%$. Utilizamos o programa Microsoft ${ }^{\circledR}$ Excel 2007 para arquivamento dos dados e o programa Statistical Package for the Social Sciences (SPSS) versão 13.0 para Windows para as análises estatísticas.

O projeto foi aprovado pelo Comitê de Ética em Pesquisa da Irmandade da Santa Casa de Misericórdia de São Paulo com o número do projeto 298/11. As pacientes do estudo assinaram um Termo de Consentimento Livre e Esclarecido.

\section{Resultados}

Foram avaliadas as biópsias de 40 pacientes, com média de idade de 36,3 anos e desvio padrão (DP) 5,7. Ao cruzar os dados com o questionário SF-36, previamente aplicado, apenas lâminas de 32 casos puderam ser correlacionadas aos resultados.

Tabela 1. Distribuição da média e desvio padrão das variáveis idade, peso, índice de massa corporal, paridade e raça em mulheres portadoras de endometriose profunda intestinal submetidas a tratamento cirúrgico laparoscópico

\begin{tabular}{lc}
\hline Variável & Média \pm DP \\
\hline Idade (anos) & $36,3 \pm 5,7$ \\
Peso $(\mathrm{kg})$ & $62,5 \pm 9,1$ \\
IMC $\left(\mathrm{kg}^{2} / \mathrm{m}\right)$ & $22,8 \pm 2,7$ \\
\hline \multicolumn{2}{c}{ n $(\%)$} \\
\hline Paridade & \\
0 & $29(90,6)$ \\
1 & $2(6,3)$ \\
2 & $1(3,1)$ \\
Raça & \\
Branca & $25(78,1)$ \\
Negra & $6(18,8)$ \\
Oriental & $1(3,1)$ \\
\hline
\end{tabular}

IMC: índice de massa corporal; DP: desvio padrão.
Observamos entre as 32 pacientes que as biópsias foram realizadas nas respectivas localizações e com os tipos histológicos descritos a seguir: no peritônio, 63\% GI e 22\% GM; no intestino, $19 \%$ GI e $24 \%$ GM; e no ligamento uterossacro, $41 \%$ GI e $35 \%$ GM.

Dois tipos histológicos fizeram parte de nossa estatística, GM e GI, por serem as mais frequentes. O tipo glandular bem diferenciado e o estromal não foram caracterizados na amostra em quantidade suficiente para uma análise estatística significante, finalizamos com uma amostra de 32 pacientes.

Buscando estabelecer associação entre o tipo histológico e a qualidade de vida das pacientes, realizou-se a análise estatística dividindo-se 32 casos de acordo com a localização da lesão biopsiada.

Avaliando os casos com biópsia em peritônio (Figura 2 e Tabela 2) e fazendo uma análise comparativa entre os dois tipos histológicos (GM e GI) e a evolução em dois momentos ( 0 a 6 meses e 0 a 12 meses) foi evidenciado no D1 (capacidade vital) uma tendência dos tipos histológicos no sentido de maiores valores para o GI nos tempos 0 a 6 meses $(p=0,1)$ e 0 a 12 meses $(p=0,1)$. No D3 e no D7 houve tendência à melhora entre as pacientes com GI no tempo 0 a 6 meses $(p=0,06-D 3 ; p=0,05-D 7)$, o que não aconteceu no tempo 0 a 12 meses $(\mathrm{p}=0,2-\mathrm{D} 3$; $\mathrm{p}=0,2-\mathrm{D} 7)$. No D3, no que se refere à evolução dos tipos histológicos, foi favorável a melhora tanto entre as mulheres com GI como no GM. No D7, a evolução do GM não foi favorável a melhora nos tempos 0 a 6 meses, embora tenha sido adequada no GI.

Considerando o D4, tivemos uma melhora significativa dos índices de qualidade de vida do GI no tempo 0 a 6 meses $(\mathrm{p}=0,01)$ e apenas uma tendência no tempo 0 a 12 meses $(p=0,07)$. Na evolução dos tipos histológicos foi favorável a melhora nos tipos GI e GM, em ambos os momentos.

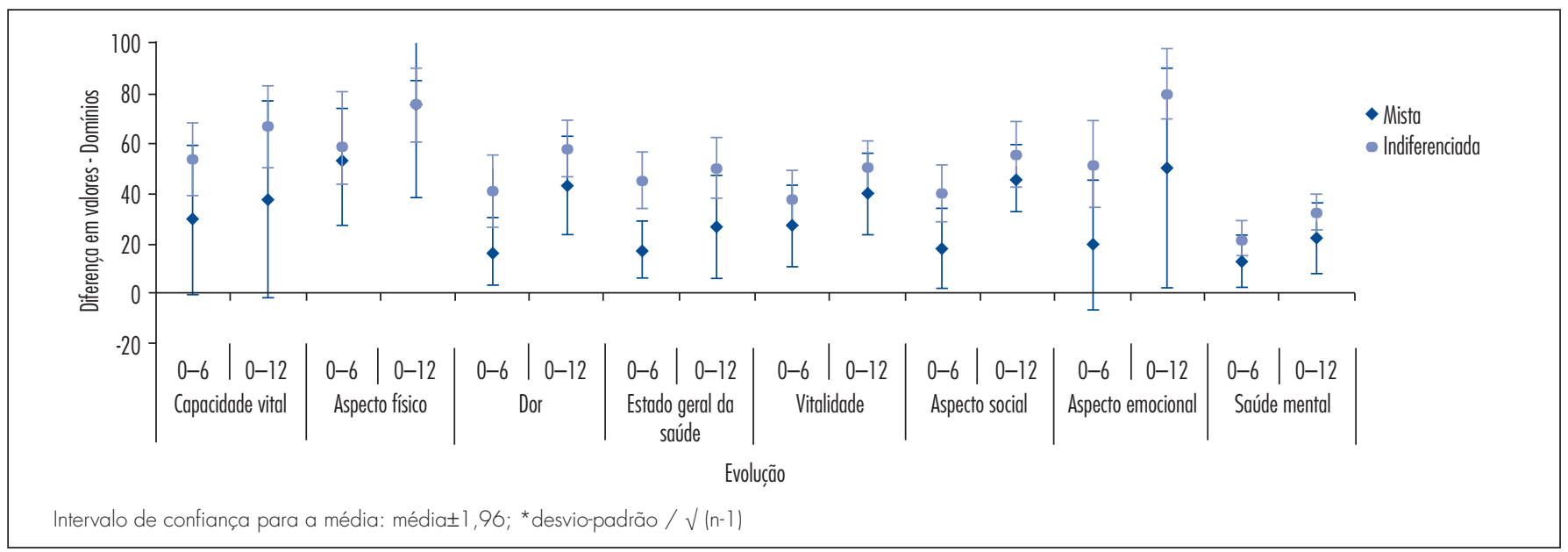

Figura 2. Análise global comparativa da média e do desvio padrão da qualidade de vida de mulheres portadoras de endometriose profunda e do grau de diferenciação histológica de biópsia de peritônio, avaliados pelo questionário SF-36 
Tabela 2. Análise global comparativa da média e do desvio padrão da qualidade de vida de mulheres portadoras de endometriose profunda e do grau de diferenciação histológica de biópsia de peritônio e intestino, e todos os domínios do questionário SF-3615

\begin{tabular}{|c|c|c|c|c|c|c|c|c|}
\hline & \multicolumn{4}{|c|}{ Peritônio } & \multicolumn{4}{|c|}{ Intestino } \\
\hline & \multicolumn{2}{|c|}{ GM } & \multicolumn{2}{|c|}{$\mathrm{GI}$} & \multicolumn{2}{|c|}{ GM } & \multicolumn{2}{|c|}{ GI } \\
\hline & $0-6$ & $0-12$ & $0-6$ & $0-12$ & $0-6$ & $0-12$ & $0-6$ & $0-12$ \\
\hline Aspectos físicos (D2) & $53,6(33,6)$ & $75,0(41,8)$ & $58,8(33,7)$ & $75,0(32,4)$ & $55,2(35,3)$ & $71,7(34,8)$ & $58,3(40,8)$ & $79,2(24,6)$ \\
\hline Dor (D3) & $16,6(16,8)$ & $43,3(22,2)$ & $41,0(31,8)$ & $57,7(26,0)$ & $35,0(33,8)$ & $55,5(28,2)$ & $37,5(32,4)$ & $55,5(26,7)$ \\
\hline Aspectos sociais (D6) & $17,9(20,2)$ & $45,8(15,1)$ & $40,0(25,5)$ & $55,6(29,9)$ & $38,0(25,7)$ & $57,6(25,2)$ & $31,3(39,3)$ & $45,8(29,2)$ \\
\hline Aspectos emocionais (D7) & $19,0(32,5)$ & $50,0(54,8)$ & $51,7(38,2)$ & $80,0(22,7)$ & $44,4(33,6)$ & $72,5(32,8)$ & $50,0(58,7)$ & $88,9(27,2)$ \\
\hline Saúde mental (D8) & $13,1(13,4)$ & & $21,8(16,2)$ & & $19,2(14,6)$ & & $24,7(24,1)$ & \\
\hline
\end{tabular}

GM: Glandular Misto; GI: Glandular; Indiferenciado; D: domínio; m: média; DP: desvio padrão; ${ }^{\star} p<0,05$.

Em relação ao D6, obtivemos uma melhora significativa do GI no tempo 0 a 6 meses $(\mathrm{p}=0,05)$, o que não acorreu no tempo 0 a 12 meses $(p=0,4)$. Na evolução dos tipos histológicos foi favorável a melhora em ambos os momentos.

Avaliando os casos com biópsia no intestino (Tabela 2), notou-se que nos D6 e D7 a evolução dos tipos histológicos não foi significante no GM no tempo 0 a 6 meses.

Nos demais domínios do SF-36 e nas outras localizações da doença (ligamento uterossacro) não houve interferência do grau histológico em relação à qualidade de vida.

A Tabela 2 representa o resultado de todos os domínios estudados relacionados aos dois tipos histológicos em pacientes com lesão no intestino.

\section{Discussão}

A endometriose profunda promove expressivo impacto sobre a qualidade de vida. Entretanto, escassos são os estudos que caracterizaram a relação entre qualidade de vida e os tipos histológicos GI e GM. Entre as 32 mulheres que caracterizamos no presente estudo, notamos predomínio do GI e GM nos sítios avaliados, resultado confirmado por estudos já realizados ${ }^{2,13}$.

Notamos que no peritônio a maior prevalência foi do tipo histológico GI, seguido pelo tipo GM, resultado que se assemelha a um estudo da literatura, mas difere de outro em que predominou o tipo estromal puro, seguido pelo $\mathrm{GI}^{12,13}$.

$\mathrm{Na}$ caracterização do tipo histológico em relação ao estádio da doença e sua profundidade, constatamos maior prevalência do tipo GI em todos os locais, seguido pelo GM. Merece ser considerado que em nossa investigação foram incluídas somente mulheres com endometriose profunda nos estádios III e IV da classificação ASRM ${ }^{6}$ e os nossos resultados coincidiram com os obtidos por outros autores $^{12,13}$.
$\mathrm{Na}$ análise da relação entre os tipos histológicos e os locais de acometimento da endometriose, notamos que o tipo GI foi identificado com maior frequência no peritônio, seguido pelo GM no mesmo local, enquanto que o padrão histológico GM foi dominante no intestino. Na literatura é mencionado que o tipo histológico GI é encontrado com frequência 2,7 vezes maior no septo reto-vaginal, em comparação com o peritônio ${ }^{12}$.

Acreditamos que a caracterização do tipo histológico possa guardar uma relação com a dor; por isso, entendemos que se essa premissa for válida, fármacos poderão ser desenvolvidos e seletivamente prescritos para atenuar a dor, a partir do conhecimento do tipo histológico e, assim, propiciar melhora da qualidade de vida. Para isso, a identificação e a resposta de agentes, a partir dos tipos histológicos, poderão ser a chave do sucesso para uma maior eficácia do fármaco e melhor qualidade de vida das portadoras de endometriose $\mathrm{e}^{20-22}$.

Ainda dentro desse raciocínio, evidências sugerem que as pacientes que melhor respondem ao tratamento cirúrgico são as do tipo histológico glandular bem diferenciado e estromal, em comparação com as do tipo GI, permitindo-nos supor que as mulheres que evoluem melhor são aquelas sem a doença indiferenciada pura ou mista ${ }^{12}$.

$\mathrm{Na}$ análise que fizemos em nosso estudo do quadro doloroso, caracterizado pelo D3 do instrumento SF-36 que avalia a qualidade de vida, apesar de não termos notado qualquer diferença da intensidade da dor entre os tipos histológicos (GI e GM), percebemos melhora no tipo GI no peritônio.

A queixa de dor isolada é difícil de ser avaliada nas mulheres com endometriose, pois o local da doença e o tipo de dor são variáveis e geram inúmeros questionamentos e arguições do quanto são fidedignos na análise final $^{9,23}$, e é por isso que no presente estudo utilizamos o SF-36, instrumento validado em todo o mundo, inclusive no Brasil ${ }^{24,25}$. 
Aplicamos o SF-36 nas portadoras de endometriose dos tipos histológicos GI e GM, que acometia peritônio, intestino e ligamento uterosacro. Diferentemente de outros estudos que caracterizaram a qualidade de vida da mulher no período pré e pós-cirúrgico ou após tratamento clínico medicamentoso ${ }^{11,26,27}$, a nossa investigação mostrou que não há comprometimento da qualidade de vida em todos os domínios do SF-36 nas portadoras da doença, de qualquer tipo histológico, no ligamento uterosacro.

Outros autores já tinham relatado melhora significativa do estado geral da saúde (D4) e do aspecto emocional (D7) após ressecção das lesões, apesar de não se ter observado diferença entre a técnica de ressecção segmentar e a nodulectomia no tratamento de endometriose intestinal ${ }^{28}$. É aceito por todos que há impacto relevante do tratamento cirúrgico da endometriose profunda ${ }^{29}$ sobre a qualidade de vida, avaliada pelo SF-36, em todos seus domínios ${ }^{18}$, especialmente entre os casos de ressecção segmentar do retossigmoide. Em nosso estudo, no entanto, ao se analisar a relação entre o tipo GM e GI no intestino, não observamos diferença significativa na melhora da qualidade de vida. $\mathrm{Na}$ evolução do tipo histológico GI no intestino também não ocorreu melhora, tanto no aspecto social como no emocional.

Observamos concordância dos nossos resultados com os da literatura no tocante ao estado geral da saúde, porém com uma diferença, quanto à localização, sendo em nosso caso limitado aos casos com lesões peritoneais. Quanto ao tipo histológico, ao analisarmos a qualidade de vida observamos melhora significativa na evolução pós-cirúrgica, limitada às mulheres com o tipo histológico GI em relação ao GM, confirmando os achados de outros autores ${ }^{28}$.

As limitações do nosso estudo incluem o número reduzido de participantes, o que nos impediu de fazer uma análise estatística da relação entre qualidade de vida e os tipos histológicos estromal e glandular bem diferenciado. Entretanto, tal fato não nos impossibilitou de caracterizar diferenças entre a evolução da qualidade de vida em função da localização das lesões e do tipo histológico.

\section{Referências}

1. Kennedy S, Bergqvist A, Chapron C, D'Hooghe T, Dunselman G, Greb R, et al. ESHRE guideline for the diagnosis and treatment of endometriosis. Hum Reprod. 2005;20(10):2698-704

2. Burney RO, Giudice LC. Pathogenesis and pathophysiology of endometriosis. Fertil Steril. 2012;98(3):511-9.

3. Darai E, Thomassin I, Barranger E, Detchev R, Cortez A, Houry $S$, et al. Feasibility and clinical outcome of laparoscopic colorectal resection for endometriosis. Am J Obstet Gynecol. 2005; 192(2):394-400.

4. Viscomi FA, Dias R, Luca L, Ihlenfeld MFK. [Correlation between laparoscopic aspects and histologic findings in peritoneal endometriotic lesions]. Rev Bras Ginecol Obstet. 2002;24(2):93-9. Portuguese.

5. Bassi MA, Podgaec S, Dias Júnior JA, Sobrado CW, D'Amico Filho $\mathrm{N}$, Abrão MS. Endometriose intestinal: uma doença benigna? Rev Assoc Med Bras. 2009;55(5):611-6.

6. Revised American Fertility Society classification of endometriosis: 1985. Fertil Steril. 1985;43(3):351-2.

7. Cornillie FJ, Oosterlynck D, Lauweryns JM, Koninckx PR. Deeply infiltrating pelvic endometriosis: histology and clinical significance. Fertil Steril. 1990;53(6):978-83.

8. Jinushi M, Arakawa A, Matsumoto T, Kumakiri J, Kitade M, Kikuchi I, et al. Histopathologic analysis of intestinal endometriosis after laparoscopic low anterior resection. J Minim Invasive Gynecol. $2011 ; 18(1): 48-53$.

9. Mabrouk M, Spagnolo E, Raimomdo D, D'Errico A, Caprara $G$, Malvi D, et al. Segmental bowel resection for colorectal endometriosis: is there a correlation between histological pattern and clinical outcomes? Hum Reprod. 2012;27(5):1314-9.
10. Novak $E$, de Lima OA. A correlative study of adenomyosis and pelvic endometriosis, with special reference to the hormonal reaction of ectopic endometrium. Am J Obstet Gynecol. 1948;56(4):634-44.

11. Schweppe KW, Wynn RM. Endocrine dependency of endometriosis: an ultrastructural study. Eur J Obstet Gynecol Reprod Biol. 1984; 17(2-3):193-208.

12. Abrão MS, Neme RM, Carvalho FM, Aldrighi JM, Pinotti JA. Histological classification of endometriosis as a predictor of response to treatment. Int J Gynaecol Obstet. 2003;82(1):31-40.

13. Kamergorodsky G, Ribeiro PA, Galvão MA, Abrão MS, Donadio N, Lemos NL, et al. Histologic classification of specimens from women affected by superficial endometriosis, deeply infiltrating endometriosis, and ovarian endometriomas. Fertil Steril. 2009;92(6):2074-7.

14. Touboul C, Ballester M, Dubernard G, Ziberman S, Thomim A, Daraï E. Long-term symptoms, quality of life, and fertility after colorectal resection for endometriosis: extended analysis of a randomized controlled trial comparing laparoscopically assisted to open surgery. Surg Endosc. 2014 [ahead of print].

15. Ciconelli RM, Ferraz MB, Santos W, Meinão I, Quaresma MR. [Brazilian-Portuguese version of the SF-36: a reliable and valid quality of life outcome measure]. Rev Bras Reumatol. 1999;39(3): 143-50. Portuguese.

16. Stull DE, Wasiak R, Kreif N, Raluy M, Colligs A, Seitz C, et al. Validation of the SF- 36 in patients with endometriosis. Qual Life Res. 2014;23(1):103-17.

17. Laas E, Zacharoupoulou C, Montanari G, Seracchioli R, Abrão MS, Bassi MA, et al. External validation of the SF-36 quality-oflife questionnaire in Italian and Brazilian populations to select patients with colorectal endometriosis for surgery. J Minim Invasive Gynecol. 2014; pii:S1553-4650(14)00308-2. 
18. Ribeiro PA, Sekula VG, Abdalla-Ribeiro HS, Rodrigues FC, Aoki T, Aldrigui JM. Impact of laparoscopic colorectal segment resection on quality of life in women with deep endometriosis: one year follow-up. Qual Life Res. 2014;23(2):639-43.

19. Ribeiro PA, Rodrigues FC, Kehdi IP, Rossini L, Abdalla HS, Donadio $\mathrm{N}$, et al. Laparoscopic resection of intestinal endometriosis: a 5-year experience. J Minim Invasive Gynecol. 2006; 13(5):442-6.

20. Carvalho L, Podgaec S, Bellodi-Privato M, Falcone T, Abrão MS. Role of eutopic endometrium in pelvic endometriosis. J Minim Invasive Gynecol. $2011 ; 18(4): 419-27$.

21. Fernandez I, Reid C, Dziurawiec S. Living with endometriosis: the perspective of male partners. J Psychosom Res. 2006;61 (4):433-8.

22. Nnoaham KE, Hummelshoj L, Webster $P$, d'Hooghe T, de Cicco Nardone F, de Cicco Nardone C, et al. Impact of endometriosis on quality of life and work productivity: a multicenter study across ten countries. Fertil Steril. $2011 ; 96(2): 366-373 . e 8$.

23. Denny E. Women's experience of endometriosis. J Adv Nurs. 2004;46(6):641-8.

24. The World Health Organization quality of life assessment (WHOQOL): position paper from the World Health Organization. Soc Sci Med. 1995;41(10): 1403-9.
25. Busija L, Pausenberger E, Haines TP, Haymes S, Buchbinder R, Osborne RH. Adult measures of general health and health-related quality of life: Medical Outcomes Study Short Form 36-ltem (SF-36) and Short Form 12-Item (SF-12) Health Surveys, Nottingham Health Profile (NHP), Sickness Impact Profile (SIP), Medical Outcomes Study Short Form 6D (SF-6D), Health Utilities Index Mark 3 (HUI3), Quality of Well-Being Scale (QWB), and Assessment of Quality of Life (AQoL). Arthritis Care Res. 2011;63(Suppl 11):S383-412.

26. Arruda MS, Petta CA, Abrão MS, Benetti-Pinto CL. Time elapsed from onset of symptoms to diagnosis of endometriosis in a cohort study of Brazilian women. Hum Reprod. 2003; 18(4):756-9.

27. Bassi MA, Podgaec S, Dias JA Jr, D’Amico Filho N, Petta CA, Abrão MS. Quality of life after segmental resection of the rectosigmoid by laparoscopy in patients with deep infiltrating endometriosis with bowel involvement. J Minim Invasive Gynecol. 2011 ; 18(6):730-3.

28. Mabrouk M, Montanari G, Guerrini M, Villa G, Solfrini S, Vicenzi $C$, et al. Does laparoscopic management of deep infiltrating endometriosis improve quality of life? A prospective study. Health Qual Life Outcomes. 2011;9:98.

29. Hidaka T, Nakashima A, Hashimoto Y, Saito S. Effects of laparoscopic radical surgery for deep endometriosis on endometriois-related pelvic pain. Minim Invasive Ther Allied Technol. 2012;21(5):355-61. 